\section{Der Tip aus der Praxis}

Immer wieder wird von niedergelassenen Augenärzten beklagt, dass Optiker die ärztliche Brillenverordnung „eigenmächtig“ abändern. Es gibt leider tatsächlich Fälle, in denen der Optiker die auf dem Rezept angegebenen Dioptrienstärken ,ïberprïft, und ohne Wissen des verordnenden Augenarztes abändert. Ein solcher Tatbestand stellt eine Verfälschung einer ärztlichen Verordnung dar und ist unstatthaft. Sehr häufig kommt es aber vor, dass der verordnende Augenarzt den Brillenverordnungsschein unvollständig ausfüllt und damit willkürlichen Auslegungen Tür und Tor öffnet.

Wie soll der Verordnungsschein korrekt ausgefüllt werden?

- Name und Geburtsdatum des Patienten und des Versicherten (Versicherungsnummer)
- Datum der Ausstellung der Verordnung (Bitten der Patienten „ohne Datum“ dürfen nicht erhört werden)

- Art des Sehbehelfes (genaue Angabe erforderlich: z.B. I Fernbrille, 1 Nahbrille, 1 Fern- und eine Nahbrille, Bifocalbrille, Gleitsichtbrille, Trifocalbrille etc.)

- Nicht ausgefüllte Felder auf dem Verordnungsschein sind deutlich zu streichen

Besondere Beachtung verdient der computergestützte Rezeptausdruck, auf dem häufig nur die Dioptrienwerte ohne Angabe der Art des Sehbehelfes aufscheinen.

Merke: sind auf dem Verordnungsschein lediglich Dioptrienangaben vorhanden, steht es bei großzügiger Auslegung dem Optiker frei, einen Sehbehelf nach seinen eigenen Vorstellungen zu fertigen.

Dr. H. Schuster 